\title{
'Moral shielding: A grounded theory of moral integrity maintenance within multidisciplinary teams'.
}

\author{
Rick Deady
}

School of Nursing and Midwifery, UCC

\section{Introduction}

On a daily basis, many health care practitioners find themselves in situations where their original aspirations to be a caring and good professional are challenged. Imagine a nurse working within a war zone where she is required, due to limited resources, to make a decision as to which of two injured soldier should have lifesaving surgery first. This is known as triage. Having made her decision, she discovers that the second soldier has died in the meantime. She knew that the right decision was for both soldiers to receive the surgery; however, the circumstances prevented this. The emotional aftermath of such a situation has been reported to lead to a debilitating condition known as 'moral distress,' where the victim feels immense guilt, shame and even anger for not acting in a manner that maintains their sense of moral integrity. Fortunately, few professionals have to make such definitive life-saving decisions. Nevertheless, many clinical decisions require some degree of commitment to adhere to the 'right' moral course of action, and in so doing many professionals draw on their own personal and professional beliefs and values in order to arrive at a decision with which their conscience is agreeable. However, current policies and directives within the health care arena dictate that such decisions should be multidisciplinary, drawing on many diverse perspectives that are influenced by personal and discipline specific values and beliefs. Nevertheless, professionals do not always agree on what constitutes a good clinical decision. In essence, the decision-making process becomes a democratic/consensus approach. So, what happens to the practitioner who does not agree with the consensus decision of the multidisciplinary team?

\section{Background}

The concept of moral distress (knowing the right thing to do but not acting) is a relatively new phenomenon under investigation within the health services, and has attracted much attention from a variety of disciplines, e.g., nursing, medicine, pharmacy, psychology and social work. Earlier studies have concentrated on situations of high intensity, such as the 
triage event above. Others have included perinatal and critical care. The majority of these studies have taken a discipline-specific approach, e.g., nurses, doctors, pharmacists etc., in their attempt to describe the properties of moral distress. Recent studies, however, have demonstrated that moral distress can also occur in less high intensity and critical environs, as well as suggesting a multidisciplinary element within the process involved in its development.

In particular, disciplines practicing within acute psychiatric environments have shown the presence of moral distress and highlighted the complex processes involved in its development. However, not all practitioners develop moral distress as a result of being involved in the same clinical decision-making process. Whilst the process of moral distress has received some considerable attention, there has been little focus on why some practitioners do not develop it. This study has identified the process of 'moral shielding' as a potential mechanism for protecting the individual's sense of moral integrity when working within a multidisciplinary environment, in the absence of moral distress.

\section{Method}

In order to avoid replication of previous studies on moral distress, I adopted a grounded theory methodology. This approach require me to enter the field of study anew, and immerse myself in the environment under investigation, in this way, I interviewed and observed practitioners in their natural environment and gained an understanding of the problems that they encounter and how they resolve them. Each interview and observation was processed as if it is a micro study. After each session I postulated a theory as to what was happening and this theory was then tested at the next interview or observation. This process continued until the evolving theory was proven at each subsequent interview, thus grounding the theory in the real world. For example, if the practice of ducking a blow is observed in all candidates being struck, then 'ducking' becomes the process of avoiding being hit. However, if some candidates swerve to avoid the blow, then 'ducking and swerving' becomes the process and the question then becomes why some duck and some swerve, hence the need to interview. In this way, the substantive theory (initial theory based on the current data collected) of moral shielding was developed. To see if this theory exists elsewhere in the world, I will be undertaking a review of the current literature associated with this area; this is the current position of the investigation.

The current findings were arrived at by interviewing thirty two practitioners involved in multidisciplinary teams within the mental health services, together with, numerous observations at clinical group meetings and projects. Moral shielding is the process of thinking and feeling in a manner that shields the self from critical commentary in order to preserve ones sense of being a person of integrity. This internal construct is externally projected in behaviours that portray the individual as a moral practitioner; this mechanism 
includes the practises of moral abdication, moral hinting, moral gauging and pseudocompliance. These practices are contingent upon the individual's ability to recognise a condition as morally wrong and the significance of that event.

It was found that, due to individual standards and discipline specific perspectives, practitioners within the same environment may not necessarily view or recognise the same situation as problematic. For example, practitioners often disagreed on the degree of risk involved in discharging a client. In addition, whilst practitioners may recognise a risk exists, unless it had potential to cause serious harm, they may not act.

Choosing not to act was premised on a number of other factors, one of which was 'moral ownership'. If it was possible to relinquish all ownership of a problem then this action was taken (primary abdication). Here, the practitioner makes the decision that the problem belongs to another and, as such, they are excused any moral implications for not acting. If, however, they are unable to relinquish all moral ownership, they may choose to share it (secondary abdication). In this instance, the practitioner demonstrates that they are not wholly responsible and that others are also implicated and have a responsibility, thus sharing burden. Practitioners who suffered moral distress were found to have taken full responsibility for the event. To cause extreme anguish for the practitioner, however the event needed to be significant, e.g., a client committing suicide due to the practitioner's inaction (tertiary abdication). Therefore, relinquishing or sharing a moral burden shields practitioners from personal responsibility and potential criticism.

Practitioners working in an environment where it was felt that open and genuine dialogue was not encouraged or in which they felt vulnerable to having their views attacked were inclined to practice moral hinting. There were wide variations in moral hinting, from subtle enquiries to conference presentations, but the more common approaches involved making an enquiry in order to gain an insight upon another's opinion, e.g., 'how do you feel about', 'would it be ok to', 'so you are happy if we'. Moral hinting allowed the practitioner to 'test the water' of opinion, rather than making a definitive stance that signalled their own moral position. Making an overt stance on a perceived wrong (speaking your mind) was viewed as professionally risky and potentially dangerous for future relationships within the multidisciplinary team. Moral hinting shields the individual from such attacks and allows them to gauge the moral climate.

Gauging the moral climate of the multidisciplinary team was seen as a necessary strategy in order to make decisions on how to act in situations of disagreement. If a practitioner morally hinted as to their position but perceived that they were being ignored (morally shunned) they would retreat from the decision-making process. During this retreat, they would continue to gauge the moral climate of the multidisciplinary team for open and genuine dialogue that would allow them to share and discuss their concerns. Moral gauging also allows the practitioner to monitor any deterioration in the situation that may signal their need to act due to the event becoming potentially significant. 
Not engaging in multidisciplinary team decision-making processes could be considered unprofessional, and so in order to avoid this accusation practitioners acted in a way that suggested that they were in agreement with decisions whilst at the same time, disagreeing internally (pseudo-compliance), and when the opportunity allowed (out of the sight of other practitioners) they practiced in their preferred manner. Long-term pseudo-compliance has the potential to develop a clinical environment for moral stagnation where significant clinical problems remain unresolved and stagnant.

\section{Conclusion}

It is hoped that the outcome of this study will be a valuable resource to professional groups wishing to achieve genuine multidisciplinary practice that aids in good clinical decisionmaking. By understanding the processes involved in moral shielding, practitioners will gain an insight into their own practice and the practice of other disciplines, as well as, recognising the need to be sensitive to the values and beliefs of fellow colleagues.

In this regard, it is clear that practitioners have demonstrated within the study their need to be heard in clinically challenging situations, as otherwise they will engage in practices to maintain their own personal integrity that are not necessarily beneficial to good multidisciplinary decision-making or client care. Due to factors outside of the practitioner's control, such as power inequality and multiple disciplinary perspectives, the heath care arena may not necessarily be the optimal environment for the discussion of clinically challenging situations. It is, perhaps, an opportune time to consider the need for an ethics ombudsman where staff can seek advice and support for their views in a supportive and non-threatening environment that removes their personal responsibility for resolving the situation. In this way, attacks on personal belief systems are removed and practitioners can avoid developing moral distress through abdication of part of their moral ownership to another in a more constructive mechanism that has the potential to resolve the clinical problem.

Rick Deady is a College Lecturer within the Catherine McAuley School of Nursing and Midwifery and a part-time Doctoral Student. His main areas of interest are Psychiatric Nursing, Multidisciplinary Practice and Forensic Psychiatry. I would like to acknowledge the support and guidance of my supervisors Dr. Tom Andrews and Dr. Joan McCarthy in the production of this work. 